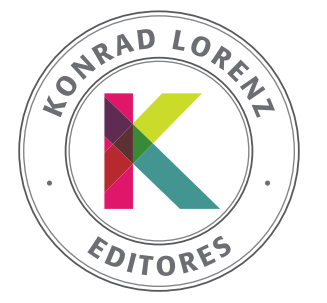

\title{
Aspectos de la cultura organizacional y su relación con la disposición al cambio organizacional
}

\author{
Juan Javier Vesga Rodríguez ${ }^{\mathrm{a}}$, Mónica García Rubiano ${ }^{\mathrm{a}}$, Carlos Forero Aponte ${ }^{\mathrm{a}}$, \\ María Constanza Aguilar Bustamante ${ }^{b *}$, Jairo Ángel Jaramillo ${ }^{b}$ Eliana Quiroz González \\ Esther Julia Castaño González ${ }^{c}$, Verónica Andrade Jaramillod y María Alejandra Gómez Vélez ${ }^{d}$
}

\author{
a Universidad Católica de Colombia, Bogotá, Colombia

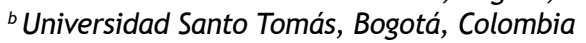 \\ c Universidad Católica de Pereira, Pereira, Colombia \\ 'Universidad Pontificia Bolivariana, Medellín, Colombia
}

Recibido el 18 de febrero de 2020; aceptado el 21 de junio de 2020

\author{
PALABRAS CLAVE \\ Cultura organizacional, \\ cambio organizacional, \\ disposición al cambio \\ organizacional, \\ psicología organizacional, \\ gestión de \\ recursos humanos
}

\begin{abstract}
Resumen El objetivo de esta investigación fue determinar la relación entre la cultura organizacional y la disposición al cambio organizacional. Se trata de una investigación empírica que, de acuerdo con el objetivo de investigación, responde a una estrategia asociativa, en un estudio predictivo desarrollado bajo un diseño correlacional simple. La muestra estuvo compuesta por 558 participantes de cuatro entidades de educación superior. Para la evaluación de las variables se emplearon la adaptación para Colombia del OCAI (Organizational Culture Assessment Instrument) y la prueba Disposición al Cambio Organizacional. Los resultados mostraron relaciones significativas entre los factores y los tipos de cultura organizacional con las macro y microdimensiones de la disposición al cambio organizacional. También se encontraron diferencias significativas entre las instituciones en las dimensiones grupal y organizacional de la disposición al cambio, en el factor organizacional y en el tipo de cultura jerárquica. Se evidencia cómo la cultura organizacional se relaciona particularmente con aspectos organizacionales y la comunicación empresarial.

(C) 2020 Fundación Universitaria Konrad Lorenz. Este es un artículo Open Access bajo la licencia CC BY-NC-ND (http://creativecommons.org/licenses/bync-nd/4.0/).
\end{abstract}

\footnotetext{
* Autor para correspondencia.

Correo electrónico: mariaaguilar@usantotomas.edu.co
} 


\section{KEYWORDS}

Organizational culture, organizational change, disposition to organizational change, organizational psychology, human resources management
Aspects of organizational culture and its relation to the organizational change disposition

Abstract The objective of this research was to determine the relationship between aspects of organizacional culture and the disposition to organizacional change. A quantitative, nonexperimental study was carried out under a comparative design; with a sample of 558 participants from four higher education institutions. The instruments used allowed the evaluation of the organizational culture-OCAl-and the willingness to change. The results showed significant relationships between the factors and the types of organizational culture with the macro and micro dimensions of the disposition to organizational change. Significant differences were also found between the institutions in the group and organizational dimensions of the disposition to change; in the organizational factor and the type of hierarchical culture. It is evident how the organizational culture is particularly related to organizational aspects and business communication.

(c) 2020 Fundación Universitaria Konrad Lorenz. This is an open access article under the CC BYNC-ND license (http://creativecommons.org/licenses/bync-nd/4.0/).
La importancia de la imagen corporativa basada en hechos concretos con relación al entorno se manifiesta claramente en el informe global anual de tendencias de capital humano (Deloitte, 2018), el cual reporta datos de por lo menos 11000 encuestas realizadas a gerentes de empresas de 140 países y a cerca de 150 líderes empresariales de Colombia. En el informe se planteó que "en muchos sentidos el capital social, está logrando un nuevo estatus junto al capital financiero y físico en valor (p. 2)". A fin de comprender mejor este aspecto se debe tener en cuenta la identidad corporativa y el compromiso de los trabajadores en relación con la cultura y la adecuada gestión del cambio presentes en cualquier tipo de organización.

Estos últimos se constituyeron en uno de los principales intereses de los gerentes de empresas en todo el mundo, ya que los estudios se enfocaron en las relaciones entre la cultura y el compromiso como aspectos claves del empleado (Deloitte, 2017). Se encontró evidencia respecto a cómo la habilidad de las organizaciones para enfrentar los problemas de compromiso y cultura se había reducido en un $14 \%$ en relación con el 2016 . Esto refleja la complejidad creciente del ambiente en el mundo laboral y da cuenta de la necesidad de desarrollar conocimiento científico que sirva para orientar a académicos y empresarios en líneas de trabajo que permitan mejorar la comprensión e intervención en estos aspectos.

\section{Cultura organizacional}

El concepto de cultura organizacional surge desde la antropología y la sociología, disciplinas en las que se ha estudiado como una manera de describir y entender las diferencias en valores esenciales que caracterizan colectivos sociales (Schneider \& Barbera, 2014). En la actualidad se entiende como una parte del contexto organizacional interno y externo (Gonzáles-Limas, Bastidas-Jurado, FigueroaChaves, Zambrano-Guerrero \& Matabanchoy-Tulcán, 2018).

En la literatura especializada en gestión empresarial se evidenció el interés de los investigadores en el éxito de las empresas japonesas y la posterior emergencia de este país como potencia mundial, con lo que se logra mostrar la relevancia del papel de la cultura en el desempeño organizacional (Denison, 1996; Gómez, Sánchez \& Del Almo, 2008; Morgan, 1998). No obstante, la primera conceptuali- zación la realizó Pettigrew (1979), quien definió la cultura como "el sistema de significados aceptados pública y colectivamente que operan para un determinado grupo en un momento dado" (p. 574).

En la investigación sobre cultura organizacional se ha considerado como uno de los modelos más influyentes el denominado marco de valores de competencia (CVF, por sus siglas en inglés) (Yu \& Wu, 2009). Este, inicialmente, se empleó para investigaciones concentradas en la identificación de indicadores sobre efectividad organizacional (Quinn \& Rohrbaugh, 1983), lo que llevó a la generación de grandes controversias. A fin de responder a esta problemática, Quinn y Rohrbaugh (1983) desarrollaron una investigación en la que identificaron tres dimensiones de valor: internaexterna, flexibilidad-control y medios-fines (esta última se integró en las dos primeras). A partir de estas dos dimensiones establecieron cuatro modelos, cada uno con diferentes criterios respecto a la efectividad humana; estos fueron denominados tipos de cultura organizacional (Yu \& Wu, 2009). El CVF propone la existencia de valores en competencia en el funcionamiento de las organizaciones (flexibilidad y discreción vs. estabilidad y control en el eje vertical, y énfasis interno e integración vs. énfasis externo y diferenciación en el eje horizontal), lo cual da origen a los tipos de cultura mencionados.

Los estudios a partir del CVF fueron retomados por Cameron y Quinn (2006), quienes identificaron cuatro tipos de cultura (clan, adhocrática, de mercado y jerárquica) y desarrollaron un instrumento para evaluar la cultura organizacional (Organizational Culture Assessment Instrument-OCAl) (véase la figura 1). Para estos autores, la cultura hace referencia a los aspectos implícitos $-\mathrm{y}$ a menudo indiscernibles- de las organizaciones, e incluye valores centrales e interpretaciones consensuadas acerca de cómo son las cosas.

De acuerdo con Yu y Wu (2009), el marco de valores de competencia es uno de los más empleados en la investigación sobre cultura organizacional y la prueba asociada (OCAI) tiene amplia validez y confiabilidad en comparación con otras escalas.

Los cuatro tipos de cultura establecidos por Cameron y Quinn (2006) presentan características que permiten identificarlas de forma adecuada; no se consideran independientes la una de la otra $y$, por tanto, lo que se espera 


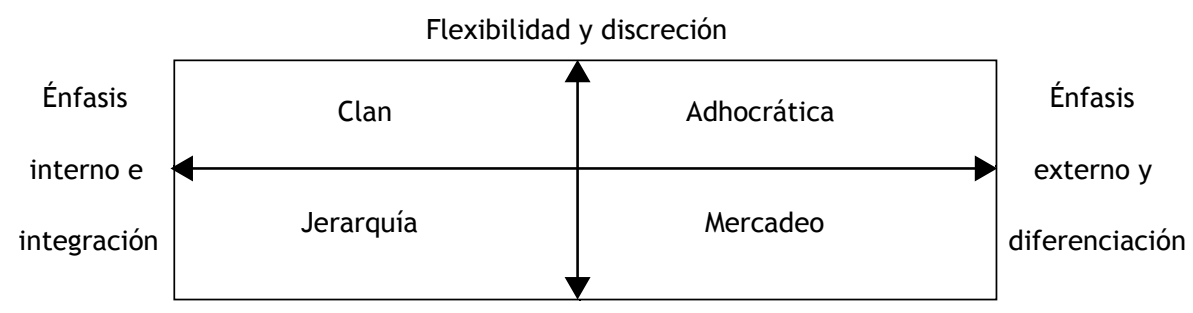

Estabilidad y control

Figura 1. Identificación de valores en competencia en la organización y tipos de cultura organizacional. Fuente: Cameron y Quinn (2006, p. 35)

es la predominancia de características de un tipo frente a los otros. En la cultura clan prevalecen el trabajo en equipo, el apoyo y la orientación de los líderes, quienes actúan como mentores, de modo que prevalecen la lealtad y la tradición. En la cultura adhocrática, la organización se concibe como dinámica, cambiante y altamente flexible, innovadora y de avanzada. La cultura de mercadeo está orientada hacia los resultados y evidencia organizaciones sumamente competitivas, con líderes altamente exigentes. Por último, la cultura jerárquica mantiene una estructura rígida, con políticas y reglas claramente establecidas, de modo que es muy importante el cumplimiento estricto de las normas y el orden jerárquico (Cameron \& Quinn, 2006).

El estudio realizado por Haffar, Al-Karaghouli y Ghoneim (2013) examinó la influencia de cuatro tipos de cultura organizacional del OCAl sobre el nivel de preparación individual para el cambio. Se identificó el tipo de cultura y las características de esas organizaciones. Los resultados de los análisis indicaron que existe mayor correlación entre los componentes de la preparación individual para el cambio y los tipos de cultura adhocrática; la investigación tuvo como fin brindar una perceptiva holística, más que centrarse en el impacto de los tipos de cultura organizacional sobre la preparación individual para el cambio.

La cultura organizacional constituye el espacio psicosocial en el que se llevan a cabo todas las actividades en la gestión de las organizaciones. Se ha evidenciado su impacto en los individuos y se considera dicha variable un importante predictor del desempeño organizacional (Cameron \& Quinn, 2006). Así, cualquier proceso de cambio debe involucrar el estudio de la cultura organizacional (Vesga, 2013). No hay duda de que las condiciones del mundo actual obligan a enfrentar de manera permanente transformaciones drásticas y aceleradas en todas las esferas de la vida humana y, en particular, en el campo de los negocios. En este orden de ideas, se hipotetiza que la cultura organizacional tiene importantes implicaciones en la disposición al cambio por parte de los miembros de una organización. La cultura organizacional es un recurso para lograr los objetivos de la organización, hace referencia a la forma como se comportan las personas y permite diferenciar a una organización de otras (Chávez-Hernández, 2020).

\section{Cambio organizacional}

La concepción del cambio organizacional, al igual que la naturaleza del concepto, es dinámica; es decir, a través del tiempo ha sufrido modificaciones dependiendo del momento histórico de análisis, así como del contexto organizacional predominante (Roskin, 1986).
De acuerdo con Grant, Michelson, Oswick y Wailes (2005), el cambio organizacional es la transformación a la cual está sujeta una organización y que puede causar diferente impacto en la cultura. Según estos autores, se trata de un proceso flexible, combinado y de doble entrada. Es un proceso multiperspectivo en el cual se contempla la construcción de una realidad social, tiene un significado de negociación (que da forma e influye en las actitudes y los comportamientos de las personas) y, finalmente, es un fenómeno intertextual en el que múltiples contextos y situaciones prevalecen en el ambiente interno y externo. En fin, es cualquier modificación significativa en los elementos, las reglas, las condiciones o las finalidades de un sistema o de cualquier parte de este (Martínez-Moreno, 2014).

El cambio organizacional es esa transformación de los procesos y las actividades desarrollados por el individuo en la organización, con lo cual se genera un impacto sociocultural significativo para promover un adecuado desempeño laboral que incremente la satisfacción tanto del individuo como del grupo y se logra mayor rentabilidad en la organización. Cuando se presenta un cambio dentro de la organización, todos los integrantes reciben una información básica sobre los cambios que se planean implementar; en ese momento se generan los primeros cuestionamientos y el análisis de cómo estos cambios pueden afectar su situación laboral actual. En este sentido, Rodríguez y Mladinic (2016), en su investigación, ponen en evidencia la importancia de la información en los procesos de cambio y el efecto de la ambivalencia actitudinal frente al cambio organizacional, puesto que los trabajadores expuestos a información inconsistente presentan mayores niveles de ambivalencia. Asimismo, se evidencia el papel moderador de la ambivalencia en la relación entre la actitud y la intención conductual del trabajador. En esencia, a fin de que el cambio sea significativo, la organización debe tener la capacidad de implementarlo de tal forma que se logre cumplir los resultados propuestos sin que esto afecte de forma negativa al trabajador (Amis \& Aïssaoui, 2013).

Otra mirada para el análisis del cambio organizacional es el de las disciplinas inmersas en este: (a) la psicología social, (b) la sociología y (c) la economía (Jacobs, Witteloostuijn \& Zeyse, 2012). Estas disciplinas se enfocan en el lugar de trabajo, en los aspectos individuales de las organizaciones y en su supervivencia. Además, se subdividen en diferentes escuelas de pensamiento, lo que da lugar a varias teorías que consideran aspectos tales como los efectos de contenidos específicos del entorno, la necesidad de flexibilidad, la capacidad de adaptación y los efectos del proceso. Los aspectos mencionados llevan a tener en cuenta teorías 
integradas del cambio organizacional que consideran tanto micro como macrosistemas, es decir, el mundo interior y exterior de las organizaciones.

Es necesario observar los principales aspectos de la naturaleza del cambio organizacional: (a) es una estrategia arriesgada, gracias a su estrecha relación con la violación de los valores culturales básicos y la identidad de la organización; (b) necesita un enfoque que muestre características específicas de la organización, identificando el ambiente interno y externo, ya que estos son necesarios en el propósito de realizar adecuados procesos de cambio organizacional; y (c) la influencia de las diferencias culturales e institucionales entre los países, esto debido a la diversidad cultural del mundo que exige una mirada amplia para abordar el cambio en las organizaciones.

Otro aspecto relacionado con la naturaleza del cambio es la disposición a él, considerado como un factor clave que explica las respuestas a los eventos organizacionales (Smollan, Matheny \& Sayers, 2010). El abordaje disposicional implica la tendencia de las personas a sentir y actuar en relación con su contexto laboral, independientemente de la naturaleza y las características de su trabajo (Weiss \& Cropanzano, 1996). En este artículo se adopta el análisis de la posición de los sujetos frente al cambio desde la perspectiva de la disposición.

El estudio realizado por Villa, Pons y Obando (2018), con una muestra de 63 colaboradores utilizando una metodología de diagnóstico de cambio y cultura organizacional para el desarrollo de la gestión de calidad en instituciones de educación superior (IES), demostró la factibilidad de su aplicación. Se identificaron las barreras culturales propias de este tipo de entidades, así como la aplicación de los planes de mejora para las variables sociales incrementó el compromiso y la participación de los participantes en relación con la certificación de la calidad y la acreditación.

Por otra parte, en la revisión realizada por Gutiérrez (2010) se señala que la prospectiva, el cambio y la cultura organizacional son aspectos que se relacionan desde una interacción teórica- conceptual. Esto debido a que son aspectos que aluden a procesos desarrollados en las IES, pero que requieren de estrategias que permitan potencializar los procedimientos operativos de este tipo de organizaciones, así como considerar la importancia del capital intelectual y humano, lo cual implica creencias y valores como factor de construcción, lo cual puede influir en la direccionalidad de estas organizaciones en los procesos de cambio.

De acuerdo con lo anterior, la pregunta de investigación es: ¿cuál es la relación entre los aspectos de la cultura organizacional y la disposición al cambio organizacional? Como objetivo general se plantea determinar la relación entre los aspectos de la cultura organizacional y la disposición al cambio organizacional. Los objetivos específicos son: (a) caracterizar la cultura organizacional y la disposición al cambio en las organizaciones participantes, y (b) analizar la relación entre los aspectos de la cultura organizacional y la disposición al cambio organizacional.

Las hipótesis que se plantearon fueron: (a) la cultura organizacional tiene relación con la disposición al cambio organizacional; y (b) los aspectos de la cultura organizacional tienen relación con los aspectos individual, grupal y organizacional de la disposición al cambio organizacional.

\section{Método}

\section{Diseño}

De acuerdo con Ato, López y Benavente (2013), es una investigación empírica realizada con una estrategia asociativa en un estudio predictivo con un diseño correlacional simple, el cual se caracteriza porque busca conocer la relación funcional entre dos o más variables, a fin de predecir el comportamiento de los grupos y que, en este caso, corresponden a cultura y disposición al cambio organizacional de personas que pertenecen al mismo sector tanto laboral como cultural.

\section{Participantes}

Dadas las condiciones de la investigación, se realizó un muestreo no probabilístico por cuotas. Se consideró un error de muestreo del $5 \%$, con un nivel de confianza del $99 \%$ en una población total de 3016 personas y con una muestra esperada de 545, la cual fue superada. Las condiciones de inclusión fueron que se tratara de personas mayores de edad, los participantes fueran funcionarios (docentes o administrativos) de las IES participantes, que se aceptara el consentimiento informado, y responder el 95\%, por lo menos, de las preguntas de los cuestionarios. Los criterios de exclusión fueron: personas menores de edad, que no se aceptara el consentimiento informado y que no se completara de forma adecuada la información sociodemográfica o de los cuestionarios.

Finalmente, la muestra estuvo constituida por 561 empleados de cuatro instituciones de educación superior (IES) de Colombia. Se retiraron los datos de tres participantes por no contar con el mínimo de calidad exigido para el procesamiento de la información. Los 558 participantes fueron 318 hombres y 240 mujeres que tenían entre 20 y 83 años $(\bar{x}=42.73 ; S=10.97)$; el $71.3 \%$ con nivel de educación de posgrado, el $13.4 \%$ de pregrado, el $12.2 \%$ eran tecnólogos y el $3 \%$ con nivel de secundaria; el $41.2 \%$ eran casados, el $34.8 \%$ solteros, el $13.1 \%$ vivía en unión libre y el $10.9 \%$ eran divorciados o separados; todos pertenecientes tanto al sector administrativo como docente de las IES.

En cuanto a la distribución de los participantes, 212 personas eran de la IES1, 61 de la IES 2, 209 de la IES 3 y 70 de la IES 4. El tiempo de ingreso laboral en las IES fluctuaba entre seis meses y 41 años; el tipo de contratación, en el $64.34 \%$ de los casos, era a término fijo, en el $33.15 \%$ a término indefinido, en el $1.43 \%$ por prestación de servicios y en un $1.08 \%$ por obra/labor.

\section{Instrumentos}

A fin de medir la variable criterio -disposición al cambio organizacional- se empleó el instrumento Disposición al cambio organizacional, diseñado por García y Forero (2016). El instrumento está constituido por tres macrodimensiones: (a) individual, (b) grupal y (c) organizacional, así como por nueve subdimensiones que las componen: (a) aceptación, (b) motivación, (c) comunicación, (d) liderazgo, (e) conocimiento, (f) clima y (g) cultura. La prueba general demostró 
ajuste en cuanto a la unidimensionalidad, pues su varianza explicada es igual a $25.5 \%$, por lo cual se evidencia ajuste en los parámetros de dimensionalidad. En cuanto a los ítems, a nivel estadístico la prueba muestra el equilibrio esperado al tener un measure de 0.00 , así como en las medidas de infit y outfit, las cuales son de 1.00 y 1.13 , respectivamente, y un modelo de error de 0.04. La confiabilidad que muestra este aspecto está ajustada y corresponde a 0.94. Las calificaciones de cada dimensión y subdimensión se obtienen mediante promedios, por lo cual fluctúan entre valores mínimo de uno (1) y máximo de seis (6).

A fin de medir la variable predictora -cultura organizacional- se utilizó el instrumento diseñado por Cameron y Quinn (2006): Organizational Culture Assessment Instrument (OCAl). Este lo constituyen seis dimensiones o factores: (a) características dominantes, (b) liderazgo organizacional, (c) administración de personal, (d) unión de la organización, (e) énfasis estratégico y (f) criterio de éxito; así como cuatro tipos de cultura organizacional: (a) clan, (b) adhocracia, (c) mercado y (d) jerarquía. La confiabilidad del instrumento, referida a qué tanto este mide de forma consistente los tipos de cultura, ha sido analizada en varios estudios. En el estudio realizado por Quinn y Spreitzer (1991) el coeficiente alfa de Cronbach ha sido .74 para la cultura clan, .79 para la cultura adhocrática, .73 para la cultura jerárquica y .71 para la cultura de mercadeo. En el estudio realizado por Yeung, Brockbank y Ulrich (1991) los resultados fueron: .79 para la cultura clan, .80 para la cultura adhocrática, .76 para la cultura jerárquica y .77 para la cultura de mercadeo. En un tercer estudio, realizado por Zammuto y Krakower (1991), se halló una confiabilidad de .82 para la cultura clan, .83 para la adhocrática, .78 para la cultura de mercadeo y .67 para la jerárquica (Cameron \& Quinn, 2006). Las calificaciones para los factores fluctúan entre 4 y 36 , en tanto que para los tipos de cultura los valores pueden ir desde 6 hasta 54 .

En cuanto a la validez del instrumento, Quinn y Spreitzer (1991) han encontrado evidencia para dos tipos de validez: convergente y discriminante. En cuanto a la primera, todos los coeficientes de correlación diagonal fueron estadísticamente diferentes de cero $(p<.001)$ y se obtuvieron rangos entre .212 y .515 . en cuanto a la validez discriminante; usando el coeficiente de Kendall se obtuvo un resultado de .764 $(p<.001)$ (Cameron \& Quinn, 2006). Para la realización del estudio se utilizó la adaptación cultural al español hecha por Sánchez y De la Garza (2013). Se evaluó la confiabilidad y la validez del instrumento en el contexto de las instituciones de educación superior con el alfa de Cronbach para cada tipo de cultura y se tuvieron los siguientes resultados: cultura clan .837, cultura adhocrática .706, cultura de mercadeo .873 y cultura jerárquica .707. Se solicitó el permiso de las autoras para utilizar el instrumento.

\section{Procedimiento}

Esta investigación se desarrolló en tres fases: en la primera, se procedió a la revisión de la literatura, la construcción del marco teórico y, de manera simultánea, se realizó la adaptación cultural del instrumento de cultura organizacional.

Durante la segunda fase los participantes, previa aceptación del consentimiento informado, pudieron responder en línea los dos cuestionarios. Luego, durante esta mis- ma fase se procedió a la consolidación y unificación de los registros para su análisis.

En la tercera fase se realizó el análisis de los resultados tanto a nivel descriptivo con media y desviación estándar como a nivel correlacional con el coeficiente de correlación de Spearman, previa evaluación de normalidad con el estadístico Kolmogorov Smirnov por tratarse de muestras de más de cincuenta casos. Finalmente, se empleó la prueba de Kruskal Wallis con el fin de evaluar si se presentaban diferencias entre las IES evaluadas. Los análisis se ejecutaron con el software estadístico IBM SPSS 24.0

\section{Consideraciones éticas}

Según los lineamientos de la Resolución 8430 (Ministerio de Salud, 1993), en esta investigación se tuvo en cuenta el diligenciamiento del consentimiento informado, la autorización de las instituciones para llevar a cabo la investigación y la firma del acuerdo de confidencialidad en los casos así requeridos. También se cumplió con la aprobación del proyecto por parte de las instituciones investigadoras. Se trató de una investigación sin riesgo, dado que no se realizó ninguna intervención o modificación intencionada de las variables biológicas, fisiológicas, psicológicas o sociales de los participantes.

También se tuvieron en cuenta los lineamientos éticos propuestos en la Ley 1090 de 2006, en aspectos tales como la confidencialidad de la información, el anonimato respecto a la participación de los evaluados, el uso del consentimiento informado, la libre participación, el bienestar del usuario y el respeto por el buen nombre. De igual forma, se consideraron los aspectos relacionados con el cuidado necesario en la presentación de resultados diagnósticos y demás inferencias basadas en la aplicación de pruebas, hasta tanto estén debidamente validadas y estandarizadas. Se tuvo en cuenta que no son suficientes las pruebas psicológicas, las entrevistas, las observaciones y los registros de conductas para hacer evaluaciones diagnósticas; todos estos deben hacer parte de un proceso amplio, profundo e integral y deben manejarse de manera prudente (Ministerio de la Protección Social, 2006). La investigación fue avalada por el Comité de Ética de las instituciones participantes.

\section{Resultados}

A fin de responder a los objetivos de la investigación, se realizaron análisis descriptivos y correlacionales en concordancia con las condiciones de las muestras evaluadas. Frente al primer objetivo, se puede observar en la tabla 1 que los promedios de los factores de la cultura organizacional tienden a estar por encima de una media hipotética de 18, lo cual, junto con las desviaciones que se presentan, arroja evidencia del sesgo hacia la derecha en todos los casos. Los factores cohesión organizacional y énfasis estratégico se muestran como los más altos, en tanto la cohesión organizacional es el más homogéneo.

Respecto al tipo de cultura, la evidencia muestra que predomina la jerárquica con un promedio más alto y tendencia a la homogeneidad, con un sesgo idéntico al arriba mencionado (véase la tabla 1). Al evaluar los factores y los tipos de cultura por cada una de las IES, se puede observar que 
Tabla 1 Media y desviación típica para los factores y dimensiones de las variables cultura y cambio organizacional de las IES

\begin{tabular}{|c|c|c|c|}
\hline Variables & Componentes & $\bar{x}$ & $S$ \\
\hline \multirow{10}{*}{$\begin{array}{l}\text { Disposición al cambio } \\
\text { organizacional }\end{array}$} & Dimensión individual & 4.6 & 0.9 \\
\hline & Motivación & 4.3 & 1.1 \\
\hline & Aceptación & 5.1 & 0.7 \\
\hline & Dimensión grupal & 4.5 & 0.8 \\
\hline & Comunicación & 4.3 & 0.9 \\
\hline & Liderazgo & 4.8 & 0.8 \\
\hline & $\begin{array}{l}\text { Dimensión } \\
\text { organizacional }\end{array}$ & 4.8 & 0.8 \\
\hline & Clima & 4.9 & 0.8 \\
\hline & Conocimiento & 4.4 & 1.1 \\
\hline & Cultura & 4.9 & 0.9 \\
\hline \multirow{10}{*}{ Cultura organizacional } & $\begin{array}{l}\text { Características } \\
\text { dominantes }\end{array}$ & 27.2 & 6.0 \\
\hline & $\begin{array}{l}\text { Liderazgo } \\
\text { organizacional }\end{array}$ & 27.1 & 6.4 \\
\hline & $\begin{array}{l}\text { Administración de } \\
\text { los empleados }\end{array}$ & 25.4 & 6.2 \\
\hline & $\begin{array}{l}\text { Cohesión } \\
\text { organizacional }\end{array}$ & 27.3 & 5.8 \\
\hline & Énfasis estratégico & 27.3 & 6.2 \\
\hline & Criterio de éxito & 25.2 & 6.0 \\
\hline & Tipo clan & 40.4 & 9.4 \\
\hline & Tipo adhocrática & 38.6 & 9.2 \\
\hline & Tipo de mercado & 39.7 & 8.3 \\
\hline & Tipo jerárquica & 40.7 & 8.5 \\
\hline
\end{tabular}

en la IES 1 predomina el liderazgo organizacional $(\bar{x}=27.9)$ y la cultura jerárquica $(\bar{x}=42.0)$ en la IES 2 el análisis estratégico $(\bar{x}=27.8)$ y la cultura tipo clan $(\bar{x}=40.5)$; por su parte, en la IES 3 predominan las características dominantes $(\bar{x}=27.8)$ y la cultura jerárquica $(\bar{x}=40.6)$; finalmente, en la IES 4 predomina el énfasis estratégico $(\bar{x}=26.7)$ y la cultura de mercado $(\bar{x}=38.9)$.

En cuanto a la disposición al cambio organizacional, se encuentran aspectos similares con respecto al sesgo hacia los valores positivos y una tendencia hacia la homogeneidad, particularmente en las macrodimensiones. Se puede observar que la dimensión organizacional tiene una puntuación más alta y las microdimensiones que la componen también poseen estas características; sin embargo, la microdimensión aceptación en la dimensión individual tiene el promedio más alto, con una mayor tendencia a la homogeneidad (véase la tabla 1).

Al realizar el análisis específico por cada IES en las macro y microdimensiones de la disposición al cambio organizacional, se encuentra que en la IES evaluadas la variable macro más relevante es la dimensión organizacional (IES 1 , $\bar{x}=4.9 ; \operatorname{IES} 2, \bar{x}=4.6 ; \operatorname{IES} 3, \bar{x}=4.8 ; \operatorname{IES} 4, \bar{x}=4.6$ ), aunque en la IES 2 se presenta la misma puntuación en la macrodimensión individual y la variable micro es la aceptación (IES 1, $\bar{x}=5.2$; IES 2, $\bar{x}=5.1$; IES 3, $\bar{x}=5.1$; IES 4, $\bar{x}=5.0$ ) (que corresponde al conjunto de microvariables de la dimensión individual).

Como ya se mencionó, la microdimensión que presentó los promedios más altos en todas las IES fue aceptación, la cual corresponde a la macrodimensión individual. Respecto a las demás dimensiones, en la dimensión grupal -en todos los casos- se destaca la microdimensión liderazgo y en la dimensión organizacional el clima.

Respecto al segundo objetivo, se buscó establecer las relaciones entre los factores y los tipos de cultura organizacional con las macro y microdimensiones de la disposición hacia el cambio organizacional. En primer lugar, se evaluó la forma de las distribuciones con la prueba de normalidad de Kolmogorov Smirnov, dado que la muestra poseía un tamaño superior a 50 participantes, encontrándose un comportamiento no normal en todos y cada uno de los aspectos evaluados; por tanto, se empleó la prueba de correlación de Spearman.

Como se puede observar en la tabla 2, las correlaciones entre las dimensiones de la disposición al cambio organizacional y los factores y tipos de cultura organizacional son positivas y significativas $(p<0.001)$ en todos los casos; sin embargo, se trata de correlaciones débiles en la mayor parte de los casos $(0.26<\rho<0.50)$, siendo las más bajas entre el tipo de cultura de mercado con las dimensiones motivación y aceptación, ambas componentes de la macrodimensión individual en la disposición al cambio. No obstante, se pueden observar correlaciones medias moderadas, particularmente entre el tipo de cultura clan con las macrodimensiones grupal y organizacional, y las microdimensiones que la componen; también se observan correlaciones de este tipo entre la macrodimensión grupal y sus componentes con el factor liderazgo organizacional y el tipo de cultura jerárquica.

La mayor cantidad de correlaciones medias moderadas la presenta la microdimensión conocimiento, ya que correlaciona de esta forma con todos los factores y con tres de los cuatro tipos de cultura, exceptuando la cultura de mercado. La dimensión individual, a pesar de presentar correlaciones significativas con todos los factores y tipos de cultura, solo muestra niveles de asociación bajos con dichas variables (véase la tabla 2 ).

Por otra parte, en cuanto a cultura, el factor características dominantes es el que presenta las correlaciones más bajas con cambio, siendo moderada únicamente la correlación que presenta con la microdimensión conocimiento. El factor criterio de éxito también presenta correlaciones que tienden a ser bajas (véase la tabla 2).

A pesar de presentarse correlaciones significativas entre todas las variables de disposición al cambio y cultura organizacional, es importante evaluar la fuerza de la asociación, de acuerdo con Coe y Merino (2003), ya que en este tipo de evaluaciones no resulta conveniente emplear el término tamaño del efecto, dado que no se trata de una evaluación de relaciones de causalidad.

Según Cárdenas y Arancibia (2014), los valores pueden fluctuar entre los tamaños del efecto en función del tipo de evaluación realizada y los coeficientes de determinación; según Coe y Merino (2003) no es común, bajo ciertas condiciones, encontrar valores de determinación superior 
Tabla 2 Correlación de Spearman entre las dimensiones de disposición al cambio y los factores y tipos de cultura organizacional de las IES

\begin{tabular}{|c|c|c|c|c|c|c|c|c|c|c|}
\hline & 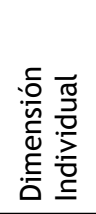 & 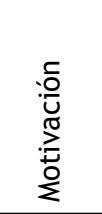 & 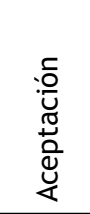 & 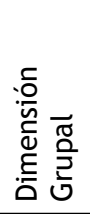 & 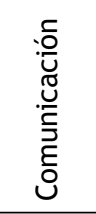 & 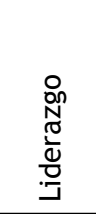 & 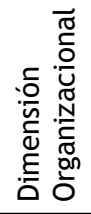 & 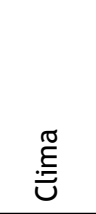 & 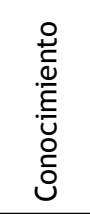 & $\frac{\widetilde{0}}{3}$ \\
\hline $\begin{array}{l}\text { Características } \\
\text { dominantes }\end{array}$ & $.342^{* *}$ & $.328^{* *}$ & $.302^{* *}$ & $.488^{* *}$ & $.496^{4 *}$ & $.432^{* *}$ & $.496^{* *}$ & $.358^{* *}$ & $.521^{* *}$ & $.425^{* *}$ \\
\hline $\begin{array}{l}\text { Liderazgo } \\
\text { organizacional }\end{array}$ & $.450^{* *}$ & $.436^{* *}$ & $.378^{* *}$ & $.576^{* *}$ & $.577^{* *}$ & $.523^{* *}$ & $.617^{* *}$ & $.451^{* *}$ & $.623^{* *}$ & $.536^{* *}$ \\
\hline $\begin{array}{l}\text { Administración } \\
\text { de los empleados }\end{array}$ & $.403^{* *}$ & $.392^{* *}$ & $.316^{* *}$ & $.522^{* *}$ & $.520^{* *}$ & $.467^{* *}$ & $.555^{* *}$ & $.407^{* * *}$ & $.578^{* *}$ & $.474^{* *}$ \\
\hline $\begin{array}{l}\text { Cohesión } \\
\text { organizacional }\end{array}$ & $.449^{* * *}$ & $.419^{* *}$ & $.420^{* *}$ & $.537^{* *}$ & $.519^{* *}$ & $.496^{* *}$ & $.582^{* *}$ & $.458^{* *}$ & $.561^{* *}$ & $.521^{* *}$ \\
\hline Énfasis estratégico & $.442^{* *}$ & $.419^{* *}$ & $.392^{* *}$ & $.544^{\star *}$ & $.528^{* *}$ & $.504^{* *}$ & $.609^{* *}$ & $.447^{\star \star}$ & $.594^{* *}$ & $.545^{* *}$ \\
\hline Criterio de éxito & $.322^{* *}$ & $.301^{* * *}$ & $.284^{* * *}$ & $.435^{* *}$ & $.421^{* *}$ & $.401^{* *}$ & $.509^{* *}$ & $.362^{* *}$ & $.519^{* *}$ & $.435^{* *}$ \\
\hline Clan & $.492^{* *}$ & $.474^{* *}$ & $.409^{\star *}$ & $.625^{* *}$ & $.614^{* *}$ & $.569^{* *}$ & $.660^{* *}$ & $.503^{* *}$ & $.658^{* *}$ & $.567^{* *}$ \\
\hline Adhocrática & $.412^{* *}$ & $.397^{\star *}$ & $.344^{* *}$ & $.541^{* *}$ & $.543^{* *}$ & $.483^{* *}$ & $.594^{* *}$ & $.424^{* * *}$ & $.604^{* *}$ & $.523^{*+}$ \\
\hline De mercado & $.301^{* * *}$ & $.274^{* *}$ & $.297^{\star \star}$ & $.406^{* *}$ & $.396^{* *}$ & $.374^{* *}$ & $.454^{\star *}$ & $.330^{* *}$ & $.470^{* * *}$ & $.383^{*+}$ \\
\hline Jerárquica & $.467^{\star *}$ & $.449^{* * *}$ & $.390^{* *}$ & $.575^{* *}$ & $.572^{* *}$ & $.519^{* *}$ & $.608^{* *}$ & $.446^{* *}$ & $.612^{* *}$ & $.532^{* *}$ \\
\hline
\end{tabular}

**. La correlación es significativa en el nivel 0.01 (bilateral).

al $30 \%$. Para esta investigación, los valores del coeficiente de determinación por encima de $30 \%$ se encuentran, fundamentalmente, en las relaciones entre la dimensión organizacional, la microdimensión comunicación y los factores liderazgo organizacional, administración de empleados, cohesión organizacional, énfasis estratégico y criterio de éxito; de igual forma, con los tipos de cultura clan, adhocrática y jerárquica, lo cual implica porciones de varianza explicada que fluctúan en esos casos entre $30.80 \%$ y $43.60 \%$ (véase la tabla 3 ).

Tabla 3 Coeficiente de determinación entre las dimensiones de disposición al cambio y los factores y tipos de cultura organizacional de las IES

\begin{tabular}{|c|c|c|c|c|c|c|c|c|c|c|}
\hline & 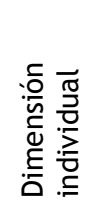 & 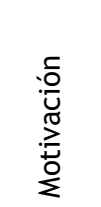 & 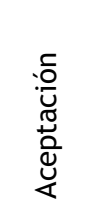 & 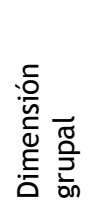 & 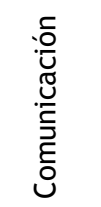 & $\begin{array}{l}\text { 옹 } \\
\text { Ñ } \\
\frac{0}{0} \\
\frac{0}{\exists}\end{array}$ & 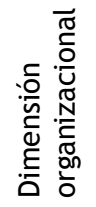 & $\stackrel{\mathscr{E}}{\Xi}$ & 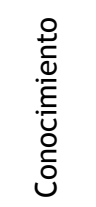 & 常 \\
\hline $\begin{array}{l}\text { Características } \\
\text { dominantes }\end{array}$ & $11.7 \%$ & $10.8 \%$ & $9.1 \%$ & $23.8 \%$ & $24.6 \%$ & $18.7 \%$ & $24.6 \%$ & $12.8 \%$ & $27.1 \%$ & $18.1 \%$ \\
\hline $\begin{array}{l}\text { Liderazgo } \\
\text { organizacional }\end{array}$ & $20.3 \%$ & $19.0 \%$ & $14.3 \%$ & $33.2 \%$ & $33.3 \%$ & $27.4 \%$ & $38.1 \%$ & $20.3 \%$ & $38.8 \%$ & $28.7 \%$ \\
\hline $\begin{array}{l}\text { Administración } \\
\text { de los empleados }\end{array}$ & $16.2 \%$ & $15.4 \%$ & $10.0 \%$ & $27.2 \%$ & $27.0 \%$ & $21.8 \%$ & $30.8 \%$ & $16.6 \%$ & $33.4 \%$ & $22.5 \%$ \\
\hline $\begin{array}{l}\text { Cohesión } \\
\text { organizacional }\end{array}$ & $20.2 \%$ & $17.6 \%$ & $17.6 \%$ & $28.8 \%$ & $26.9 \%$ & $24.6 \%$ & $33.9 \%$ & $21.0 \%$ & $31.5 \%$ & $27.1 \%$ \\
\hline Énfasis estratégico & $19.5 \%$ & $17.6 \%$ & $15.4 \%$ & $29.6 \%$ & $27.9 \%$ & $25.4 \%$ & $37.1 \%$ & $20.0 \%$ & $35.3 \%$ & $29.7 \%$ \\
\hline Criterio de éxito & $10.4 \%$ & $9.1 \%$ & $8.1 \%$ & $18.9 \%$ & $17.7 \%$ & $16.1 \%$ & $25.9 \%$ & $13.1 \%$ & $26.9 \%$ & $18.9 \%$ \\
\hline Clan & $24.2 \%$ & $22.5 \%$ & $16.7 \%$ & $39.1 \%$ & $37.7 \%$ & $32.4 \%$ & $43.6 \%$ & $25.3 \%$ & $43.3 \%$ & $32.1 \%$ \\
\hline Adhocrática & $17.0 \%$ & $15.8 \%$ & $11.8 \%$ & $29.3 \%$ & $29.5 \%$ & $23.3 \%$ & $35.3 \%$ & $18.0 \%$ & $36.5 \%$ & $27.4 \%$ \\
\hline De mercado & $9.1 \%$ & $7.5 \%$ & $8.8 \%$ & $16.5 \%$ & $15.7 \%$ & $14.0 \%$ & $20.6 \%$ & $10.9 \%$ & $22.1 \%$ & $14.7 \%$ \\
\hline Jerárquica & $21.8 \%$ & $20.2 \%$ & $15.2 \%$ & $33.1 \%$ & $32.7 \%$ & $26.9 \%$ & $37.0 \%$ & $19.9 \%$ & $37.5 \%$ & $28.3 \%$ \\
\hline
\end{tabular}


En relación con el cuarto objetivo, se calculó la normalidad de las distribuciones para cada IES. Dado que el tamaño de cada uno de los subgrupos era superior a 50 casos, se empleó la prueba de normalidad de Kolmogorov Smirnov. Los resultados evidenciaron que solo las dimensiones individual y grupal en la IES 2, la dimensión grupal en la IES 3 y la IES 4, así como el factor énfasis estratégico en la IES 4 y el tipo de cultura de mercado en la misma IES tuvieron comportamiento normal. Las demás variables en cada una de las IES presentaron un comportamiento no normal, por lo que se decide emplear la prueba de Kruskal Wallis (KS) para $k$ muestras no relacionadas. De acuerdo con Coe y Merino (2003), para este tipo de prueba estadística no existe una forma general que permita calcular el tamaño del efecto.

Al comparar las distintas variables entre las distintas instituciones mediante la prueba KS, se encontraron diferencias significativas en las dimensiones grupal (Chi-cuadrado $=16.525 ; p<0,001 \%$ ), presentándosediferencias entre las IES 1 y 4 con un nivel de confianza del 99.9) y organizacional (Chi-cuadrado $=9.553 ; p<0,023 \%$, presentándose diferencias entre las IES 1 y 4 con un nivel de confianza del $97.7 \%$ ), respectivamente. De igual forma, en el factor liderazgo organizacional (Chi-cuadrado=12.918; $p<0.005 \%$, presentándose diferencias entre las IES 1 y 4 con una confiabilidad del 99.5\%) y el tipo de cultura jerárquica (Chi-cuadrado $=10.016 ; p<0.018 \%$, presentándose diferencias entre las IES 1 y 2 con una confiabilidad del 98.2\%) (véase la tabla 4).

\section{Discusión}

En cuanto a la cultura organizacional de las IES participantes, predominan los factores cohesión organizacional y énfasis estratégico, lo cual es consistente con la preeminencia de la cultura organizacional jerárquica. Sin embargo, al analizar el comportamiento de las variables asociadas con la cultura organizacional, se puede observar una tenden- cia a los valores altos, lo que implica una predisposición en los participantes al momento de responder. Esto puede ser explicado por la presencia de un sesgo de deseabilidad social.

Esta condición se percibe en las evaluaciones realizadas en la disposición al cambio organizacional. Es un aspecto recurrente en este tipo de investigaciones, en las cuales resulta relevante evaluar la forma de contrarrestar los efectos de dichas condiciones. Se puede observar que al analizar por separado el tipo de respuesta en cada una de las IES, los resultados son similares, independientemente del tamaño de la muestra evaluada.

Las condiciones de baremación de las pruebas y la estandarización para la población colombiana son elementos que contribuyen a comprender los resultados. Es indudable que se generan sesgos en las pruebas, los cuales se pueden explicar por factores de polarización o condiciones implícitas en los pesos específicos del aporte que cada variable podría dar para la explicación del fenómeno.

En cuanto a las relaciones entre las variables, resulta interesante la disposición de la muestra a presentar correlaciones positivas en todos los casos, además de la tendencia a las correlaciones más altas con la dimensión organizacional y las más bajas en la dimensión individual. Este aspecto puede explicarse en relación a que la cultura organizacional procede de una construcción más colectiva que individual, de modo que el carácter de la organización sustenta más elementos explicativos que el grupal.

Estos resultados son respaldados por el coeficiente de determinación en las distintas relaciones que evidencian cómo la dimensión organizacional y el conocimiento aportan las varianzas explicadas más altas en los análisis; sin embargo, para efectos del alcance de la investigación y en consonancia con los criterios del análisis de este tipo de estadísticos, los valores superiores al $30 \%$ de varianza explicada son un aporte relevante a la interpretación de dichos coeficientes (Cárdenas \& Arancibia, 2014).

Tabla 4 Comparación entre las IES respecto a las variables de disposición al cambio y cultura organizacional mediante la prueba de Kruskal Wallis (KS)

\begin{tabular}{|c|c|c|c|c|}
\hline Variables & Componentes & Chi-cuadrado & gl & Sig. Asintótica \\
\hline \multirow{3}{*}{ Disposición al cambio } & Dimensión individual & 6.425 & 3 & 0.093 \\
\hline & Dimensión grupal & 16.525 & 3 & 0.001 \\
\hline & Dimensión organizacional & 9.553 & 3 & 0.023 \\
\hline \multirow{10}{*}{ Cultura organizacional } & Características dominantes & 7.781 & 3 & 0.051 \\
\hline & Liderazgo organizacional & 12.918 & 3 & 0.005 \\
\hline & Administración de los empleados & 5.579 & 3 & 0.134 \\
\hline & Cohesión organizacional & 4.057 & 3 & 0.255 \\
\hline & Énfasis estratégico & 2.756 & 3 & 0.431 \\
\hline & Criterio de éxito & 0.323 & 3 & 0.956 \\
\hline & Tipo clan & 5.155 & 3 & 0.161 \\
\hline & Tipo adhocrática & 1.938 & 3 & 0.585 \\
\hline & Tipo de mercado & 2.482 & 3 & 0.479 \\
\hline & Tipo jerárquica & 10.016 & 3 & 0.018 \\
\hline
\end{tabular}


Como era de esperarse por la tendencia de los resultados, las dimensiones grupal y organizacional mostraron evidencia de diferencias significativas entre los grupos con prevalencia del IES 1. Algo semejante ocurre con los elementos de la cultura organizacional, los cuales tienen que ver con el liderazgo y la cultura jerárquica que caracterizan a esta institución.

Esta investigación muestra cómo los significados de las variables del cambio organizacional se relacionan con la cultura, en concordancia con las definiciones de Pettigrew (1979), quien señala que la cultura se define en términos de significados aceptados pública y colectivamente. De igual manera, podría relacionarse con los planteamientos de Cameron y Quinn (2006), dado que tienen en cuenta aspectos tanto internos (los individuos) como externos (los grupos y la organización) en la identificación de los tipos de cultura organizacional. El liderazgo, el trabajo en equipo, la comunicación y el conocimiento de la organización pueden convertirse en aspectos centrales para la comprensión y la caracterización de los tipos de cultura organizacional.

En futuras investigaciones es recomendable considerar dos condiciones: la importancia de incrementar el alcance psicométrico de las pruebas empleadas - lo cual permita obtener claridad sobre las características de las variables evaluadas en la población colombiana-, y la posibilidad de establecer el alcance explicativo que puede tener el tipo de cultura y los factores que la componen sobre la disposición al cambio organizacional.

\section{Referencias}

Amis, J. M., \& Aïssaoui, R. (2013). Readiness for change: An institutional perspective. Journal of Change Management, 13(1), 69-95. https://doi.org/10.1080/14697017.2013.768435

Ato, M., López, J. J., \& Benavente, A. (2013). Un sistema de clasificación de los diseños de investigación en psicología. Anales de Psicología, 29(3), 1038-1059. https://doi.org/10.6018/analesps.29.3.178511

Cameron, K., \& Quinn, R. (2006). Diagnosing and Changing Organizational Culture. San Francisco: Jossey-Bass.

Cárdenas, M., \& Arancibia, H. (2014). Potencia estadística y cálculo del tamaño del efecto en $G^{*}$ Power: complementos a las pruebas de significación estadística y su aplicación en psicología. Salud \& Sociedad, 5(2), 210-224. https://doi.org/10.22199/ S07187475.2014.0002.00006

Coe, R., \& Merino, C. (2003). Magnitud del efecto. Una guía para investigadores y usuarios. Revista de Psicología de la PUCP, 21(1), 146-177.

Chávez-Hernández, N. (2020). Análisis cuantitativo del Modelo de Valores en Competencia en pequeñas instituciones educativas de nivel superior en el municipio mexiquense de Coacalco de Berriozábal. Ciencias Administrativas. Teoría y Praxis, 15(2), 97-112.

Deloitte. (2017). 2017 Deloitte global human capital trends. Reescribiendo las reglas para la era digital. Recuperado de https:// www2.deloitte.com/gt/es/pages/human-capital/articles/introduction-human-capital-trends-2017.html

Deloitte. (2018). El auge de la empresa social: estudio "Tendencias globales de capital humano 2018”. Resumen ejecutivo. Deloitte insights. Recuperado de https://www2.deloitte.com/co/es.ht$\mathrm{ml}$ ?icid=site_selector_co

Denison, D. (1996). What is the difference between organizational culture and organizational climate? A native's point of view on a decade of paradigm wars. Academy of Management Review, 21(3), 619-654. https://doi.org/10.5465/AMR.1996.9702100310
García, M. \& Forero, C. (2016). Calidad de vida laboral y la disposición al cambio organizacional en funcionarios de empresas de la ciudad de Bogotá-Colombia. Acta Colombiana de Psicología, 19(1), 79-90. https://doi.org/10.14718/ACP.2016.19.1.5

Gómez, A. M., Sánchez, J. C., \& Del Almo, E. A. (2008). Cultura organizacional. En F. J. Palací (Ed.), Psicología de la organización (pp. 217-244). Madrid: Pearson.

Gonzáles-Limas, W., Bastidas-Jurado, C., Figueroa-Chaves, H., Zambrano-Guerrero, C., \& Matabanchoy-Tulcán, S. (2018). Revisión sistemática de las concepciones de cultura organizacional. Universidad y Salud, 20(2), 200-201. https://doi.org/10.22267/ rus. 182002.123

Grant, D., Michelson, G., Oswick, C., \& Wailes, N. (2005). Guest editorial: Discourse and organizational change. Journal of Organizational Change Management,18(1), 6-15. https://doi. org/10.1108/09534810510579814

Gutiérrez, H. (2010). Calidad total y productividad. México. Editorial Mc Graw-Hill.

Haffar, M., Al-Karaghouli, W., \& Ghoneim, A. (2013). The mediating effect of individual readiness for change in the relationship between organizational culture and TQM implementation. Total Quality Management \& Business Excellence, 24(5-6), 693-706. https://doi.org/10.1080/14783363.2013.791112

Jacobs, G. A., Witteloostuijn, A., \& Zeyse, J. (2012). A theoretical framework of organizational change. Journal of Organizational Change Management, 26(5), 772-792. https://doi.org/10.1108/ JOCM-09-2012-0137

Martínez-Moreno, A. (2014). Cambio, desarrollo organizacional y aprendizaje de las organizaciones. En P. Gil Monte (Coord.), Manual de psicosociología aplicada al trabajo y a la prevención de los riesgos laborales (pp. 543-565). España: Pirámide.

Ministerio de Salud. (1993). Resolución No. 8430 de 1993. Ministerio de Salud. Gobierno de Colombia.

Ministerio de la Protección Social. (2006). Ley Número 1090 de 2006. Ministerio de la Protección Social. Gobierno de Colombia.

Morgan, G. (1998). Imágenes de la Organización. México, D.F.: Alfaomega.

Pettigrew, A. M. (1979). On studying organizational cultures. Administrative Science Quarterly, 24(4), 570-581.

Quinn, R., \& Rohrbaugh, J. (1983). A spatial model of effectiveness criteria: Toward a competing values approach to organizational analysis. Management Science, 29, 363-377. https://doi. org/10.1287/mnsc.29.3.363

Quinn, R. E., \& Spreitzer, G. M. (1997). The road to empowerment: Seven questions every leader should consider. Organizational Dynamics, 26(2), 37-49. https://doi.org/10.1016/S00902616(97)90004-8

Rodríguez, V., \& Mladinic, A. (2016). Ambivalencia actitudinal ante el cambio organizacional: un análisis desde el individuo en contexto laboral. Psykhe, 25(1), 1-17. https://doi.org/10.7764/ psykhe.25.1.733

Roskin, R. (1986). Corporate culture revolution: The management development imperative. Journal of Managerial Psychology, 1(2), 3-9. https://doi.org/10.1108/eb004407

Sánchez, M. I., \& Garza, M. T. de la. (2013). Cultura y estrategia en las instituciones de educación superior. Educere, 17(58), 487499.

Schneider, B., \& Barbera, K. M. (2014). Introduction: The Oxford handbook of organizational climate and culture. En B. Schneider, \& K. M. Barbera (Eds.), The Oxford handbook of organizational climate and culture (pp. 3-20). Nueva York: Oxford.

Smollan, R., Matheny, J., \& Sayers, J. (2010). Personality, affect and organisational change: A qualitative study. En N. Ashkanasy, W. Zerbe, \& C. Härtel (Eds.), Research on emotion in organizations. Emotions and Organizational Dynamism (Vol. 6, pp. 85-112). Reino Unido: Emerald Group Publishing Limited. 
Vesga, J. J. (2013). Cultura organizacional y sistemas de gestión de la calidad: una relación clave en la gestión de instituciones de educación superior. Revista Guillermo de Ockham, 11(2), 89-100.

Villa, E., Pons, R., \& Obando, M. (2018). Aplicación de una metodología de diagnóstico del cambio de la cultura organizacional en instituciones de educación superior. Espacios, 39(30), 16-24.

Weiss, H. M., \& Cropanzano, R. (1996). Affective events theory: A theoretical discussion of the structure, causes and consequences of affective experiences at work. En B. M. Staw, \& L. L. Cummings (Eds.), Research in organizational behavior: An annual series of analytical essays and critical reviews (Vol. 18 pp. 1-74). EE.UU.: Elsevier Science/JAI Press.

Yeung, A., Brockbank, J., \& Ulrich, D. (1991). Organizational culture and human resources practices: An empirical assessment. En R. W. Woodman, \& W. A. Pasmore (Eds.), Research in organizational change and development: An annual series featuring advances in theory, methodology and research (Vol 5. pp. 59-81). Greenwich: JAI Press.
Yu, T., \& Wu, N. (2009). A review of study on the competing values framework. International Journal of Business and Management, 4(7), 37-42. https://doi.org/10.5539/ijbm.v4n7p37

Zammuto, R. F., \& Krakower, J.Y. (1991). Quantitative and qualitative studies of organizational culture. En R. W. Woodman, \& W. A. Pasmore (Eds.), Research in organizational change and development: An annual series featuring advances in theory, methodology and research (Vol. 5. pp. 83-114). Greenwich, JAI Press. 